\title{
Chaotic dynamics of a Rayleigh-Duffing oscillator with periodically external and parametric excitations*
}

\author{
Liangqiang Zhou ${ }^{1, a}$, Shanshan $\mathrm{Liu}^{1, \mathrm{~b}}$ and Fangqi Chen ${ }^{1, \mathrm{c}}$ \\ Department of Mathematics, \\ ${ }^{1}$ Nanjing University of Aeronautics and Astronautics, Nanjing 210016, PR China \\ aemail: zlqrex@sina.com
}

Keywords: Rayleigh-Duffing oscillator; Chaos; Melnikov method; Controllable frequency

\begin{abstract}
Chaotic motions of a Rayleigh-Duffing oscillator with periodically external and parametric excitations are investigated rigorously. Chaos arising from intersections of homoclinic orbits is analyzed with the Melnikov method. The critical curves separating the chaotic and non-chaotic regions are obtained. The chaotic feature on the system parameters is discussed. Chaotic dynamics are also compared for the systems with a periodically external excitation or a parametric excitation. Some new dynamical phenomena including"controllable frequency" are presented. Numerical simulations verify the analytical results.
\end{abstract}

\section{Introduction}

The Rayleigh-Duffing oscillator models are widely used in physics, engineering, electronics, and many other disciplines. The nonlinear dynamics for this class of oscillators has been investigated in recent years. Using numerical and analytical approaches, nonlinear dynamics of a non-ideal Duffing-Rayleigh oscillator is studied by Felix et al [1]. Kanai and Yabuno [2] investigated creation-annihilation process of limit cycles in the Rayleigh-Duffing oscillator with negative linear damping and negative linear stiffness by the perturbation method. Qriouet and Mira [3] studied bifurcation structures related to families of fractional harmonics solutions generated by the Duffing-Rayleigh equation with a non-symmetrical periodic external force. With path integration based on the Gauss-Legendre integration scheme, Xie et al [4, 5] studied a Duffing-Rayleigh oscillator subject to harmonic and stochastic excitations. By using the asymptotic perturbation method, Siewe et al [6] studied the principal parametric resonance of a Rayleigh-Duffing oscillator with time-delayed feedback position and linear velocity terms. Mihara and Kawakami [7] studied synchronization and chaos of coupled Duffing-Rayleigh oscillators. Ma et al [8] investigated the synchronization of self-sustained Rayleigh-Duffing oscillator by the synchronization criteria based on Lyapunov direct method and the stability theory of linear time-varied systems. With the Melnikov method and Numerical methods, effects of nonlinear dissipation on the basin boundaries of a driven two-well Rayleigh-Duffing oscillator were investigated by Siewe et al [9]. Using the Melnikov method, Siewe et al [10] investigated the chaotic behavior of the Rayleigh-Duffing oscillator under a harmonic external excitation. Using the Melnikov method, Zhang and Luo [11] studied chaos of Rayleigh-Duffing like system. They also investigated the synchronization of two fractional Rayleigh Duffing-like systems with active control technology. With the Melnikov method, the effect of nonlinear dissipation on the basin boundaries of a driven two-well modified Rayleigh-Duffing oscillator was studied by Miwadinou et al [12]. With stochastic averaging method, Zhang et al [13] investigated the response of a Duffing-Rayleigh system with a fractional derivative under Gaussian white noise excitation. The effects of different system parameters and noise intensity on the response of the system are also discussed there. By using the composite cell coordinate system method, Yue et al [14] studied the global bifurcations including the crisis and metamorphosis of the Rayleigh-Duffing oscillator.

In this paper, chaotic motions of the Rayleigh-Duffing oscillator with periodically external and parametric excitations are studied analytically with the Melnikov method. The critical curves 
separating the chaotic and non-chaotic regions are plotted. The chaotic feature on the system parameters is discussed in detail and some new dynamical phenomena are presented. The phase portraits and Poincaré sections are numerically computed, which verify the analytical results.

\section{Formulation of the problem}

Consider the Rayleigh-Duffing oscillator with periodically external and parametric excitations

$$
\ddot{x}-\mu\left(1-\dot{x}^{2}\right) \dot{x}-\alpha x+\beta x^{3}=f(1+x) \cos \omega t
$$

where $\mu, \alpha$ and $\beta$ are nonlinear damping, linear and nonlinear restoring parameters, $f$ and $\omega$ are the amplitude and frequency of the excitation, respectively.

Assuming the damping $\mu$ and excitation amplitude $f$ are small, setting $\mu=\varepsilon \bar{\mu}, f=\varepsilon \bar{f}$, where $\varepsilon$ is a small parameter, then Eq.(1) can be written as

$$
\left\{\begin{array}{l}
\dot{x}=y \\
\dot{y}=\alpha x-\beta x^{3}+\varepsilon \bar{\mu}\left(1-\dot{x}^{2}\right) \dot{x}+\varepsilon \bar{f}(1+x) \cos \omega t
\end{array}\right.
$$

Consider the case of double-well potential, i.e. $\alpha>0, \beta>0$, when $\varepsilon=0$, the unperturbed system of (2) is

$$
\left\{\begin{array}{l}
\dot{x}=y \\
\dot{y}=\alpha x-\beta x^{3}
\end{array}\right.
$$

which is a planar Hamiltonian system with the Hamiltonian

$$
H(x, y)=\frac{y^{2}}{2}-\frac{\alpha x^{2}}{2}+\frac{\beta x^{4}}{4} \equiv h
$$

System (3) has one saddle $(0,0)$ and two centers $(\sqrt{\alpha / \beta}, 0)$ and $(-\sqrt{\alpha / \beta}, 0)$. There exist homoclinic orbits connecting $(0,0)$ to itself for $h=0$ with the expressions of (5), see Fig.1.

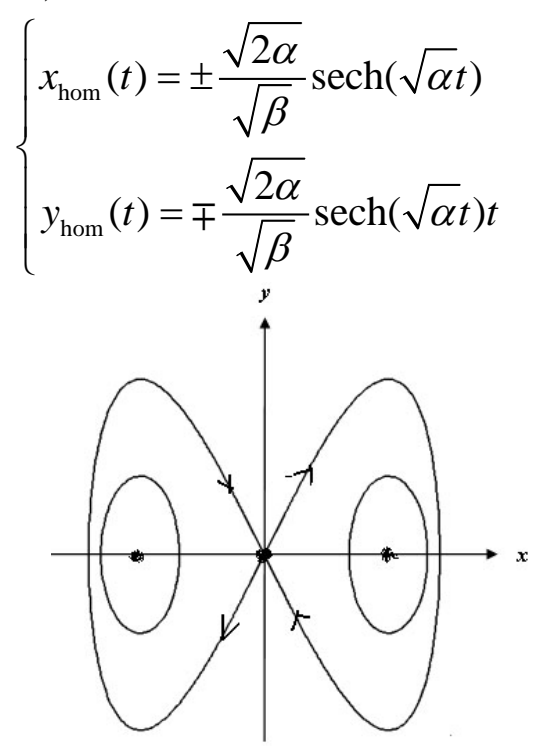

Fig.1 The phase portrait of system (3) in the case of double-well potential.

\section{Chaotic motions of the system in the case of double-well potential}

In this section, we use the Melnikov method [15] to investigate the chaotic motions of system (2). We compute the Melnikov function of system (2) along the homoclinic orbit

$$
\Gamma^{+}:\left(x_{\text {hom }}^{+}(\mathrm{t}), \mathrm{y}_{\text {hom }}^{+}(\mathrm{t})\right)=\left(\frac{\sqrt{2 \alpha}}{\sqrt{\beta}} \operatorname{sech}(\sqrt{\alpha} t),-\frac{\sqrt{2 \alpha}}{\sqrt{\beta}} \operatorname{sech}(\sqrt{\alpha} t) \tanh (\sqrt{\alpha} t)\right)
$$

as follows: 


$$
\begin{aligned}
& M^{+}\left(t_{0}\right)=\bar{\mu} \int_{-\infty}^{+\infty}\left(y_{\mathrm{hom}}^{+}(t)\right)^{2} d t-\bar{\mu} \int_{-\infty}^{+\infty}\left(y_{\mathrm{hom}}^{+}(t)\right)^{4} d t \\
& +\bar{f} \int_{-\infty}^{+\infty} y_{\mathrm{hom}}^{+}(t) \cos \omega\left(t+t_{0}\right) d t+\bar{f} \int_{-\infty}^{+\infty} x_{\mathrm{hom}}^{+}(t) y_{\mathrm{hom}}^{+}(t) \cos \omega\left(t+t_{0}\right) d t \\
& \equiv \bar{\mu}\left(I_{1}^{+}-I_{2}^{+}\right)-\bar{f}\left(I_{3}^{+}+I_{4}^{+}\right) \sin \omega t_{0}
\end{aligned}
$$

Where

$$
\begin{aligned}
& I_{1}^{+}=\int_{-\infty}^{+\infty}\left(y_{\mathrm{hom}}^{+}(t)\right)^{2} d t=\frac{2 \alpha^{2}}{\beta} \int_{-\infty}^{+\infty} \operatorname{sech}^{2}(\sqrt{\alpha t}) \tanh ^{2}(\sqrt{\alpha t}) d t=\frac{4 \alpha \sqrt{\alpha}}{3 \beta}, \\
& I_{2}^{+}=\int_{-\infty}^{+\infty}\left(y_{\mathrm{hom}}^{+}(t)\right) d t=\frac{4 \alpha^{4}}{\beta^{2}} \int_{-\infty}^{+\infty} \operatorname{sech}^{4}(\sqrt{\alpha t}) \tanh ^{4}(\sqrt{\alpha t}) d t=\frac{16 \alpha^{3} \sqrt{\alpha}}{35 \beta^{2}}, \\
& I_{3}^{+}=\int_{-\infty}^{+\infty}\left(y_{\mathrm{hom}}^{+}(t)\right) \sin \omega t d t=\sqrt{\frac{2 \alpha^{2}}{\beta}} \int_{-\infty}^{+\infty} \operatorname{sech}(\sqrt{\alpha t}) \tanh (\sqrt{\alpha t}) \sin \omega t d t=\frac{\sqrt{2 \beta} \pi \omega}{\beta} \operatorname{sech}\left(\frac{\pi \omega}{2 \sqrt{\alpha}}\right), \\
& I_{4}^{+}=\int_{-\infty}^{+\infty} x_{\text {hom }}^{+}(t) y_{\mathrm{hom}}^{+}(t) \sin \omega t d t=\frac{2 \alpha \sqrt{\alpha}}{\beta} \int_{-\infty}^{+\infty} \operatorname{sech}^{2}(\sqrt{\alpha t}) \tanh (\sqrt{\alpha t}) \sin \omega t d t=\frac{\pi \omega^{2}}{\beta \sinh \left(\frac{\pi \omega}{2 \sqrt{\alpha}}\right)} .
\end{aligned}
$$

Thus, $M^{+}\left(t_{0}\right)$ has simple zeros and chaotic motions occur if and only if

$$
\frac{\mu}{f}<\left|\frac{I_{3}^{+}+I_{4}^{+}}{I_{1}^{+}-I_{2}^{+}}\right|
$$

While the Melnikov function of system (2) along the homoclinic orbit

$$
\Gamma^{-}:\left(x_{\text {hom }}^{-}(\mathrm{t}), \mathrm{y}_{\text {hom }}^{-}(\mathrm{t})\right)=\left(-\frac{\sqrt{2 \alpha}}{\sqrt{\beta}} \operatorname{sech}(\sqrt{\alpha} t), \frac{\sqrt{2 \alpha}}{\sqrt{\beta}} \operatorname{sech}(\sqrt{\alpha} t) \tanh (\sqrt{\alpha} t)\right)
$$

is as follows:

$$
\begin{aligned}
& M^{-}\left(t_{0}\right)=\bar{\mu} \int_{-\infty}^{+\infty}\left(y_{\mathrm{hom}}^{-}(t)\right)^{2} d t-\bar{\mu} \int_{-\infty}^{+\infty}\left(y_{\mathrm{hom}}^{-}(t)\right)^{4} d t \\
& +\bar{f} \int_{-\infty}^{+\infty} y_{\mathrm{hom}}^{-}(t) \cos \omega\left(t+t_{0}\right) d t+\bar{f} \int_{-\infty}^{+\infty} x_{\mathrm{hom}}^{-}(t) y_{\mathrm{hom}}^{-}(t) \cos \omega\left(t+t_{0}\right) d t \\
& \equiv \bar{\mu}\left(I_{1}^{-}-I_{2}^{-}\right)-\bar{f}\left(I_{3}^{-}+I_{4}^{-}\right) \sin \omega t_{0}
\end{aligned}
$$

Where

$$
\begin{aligned}
& I_{1}^{-}=\int_{-\infty}^{+\infty}\left(y_{\mathrm{hom}}^{-}(t)\right)^{2} d t=I_{1}^{+}=\frac{4 \alpha \sqrt{\alpha}}{3 \beta}, \quad I_{2}^{-}=\int_{-\infty}^{+\infty}\left(y_{\mathrm{hom}}^{-}(t)\right)^{4} d t=I_{2}^{+}=\frac{16 \alpha^{3} \sqrt{\alpha}}{35 \beta^{2}}, \\
& I_{3}^{-}=\int_{-\infty}^{+\infty}\left(y_{\mathrm{hom}}^{-}(t)\right) \sin \omega t d t=-I_{3}^{+}=-\frac{\sqrt{2 \beta} \pi \omega}{\beta} \sec h\left(\frac{\pi \omega}{2 \sqrt{\alpha}}\right), \\
& I_{4}^{-}=\int_{-\infty}^{+\infty} x_{\text {hom }}^{+}(t) y_{\text {hom }}^{+}(t) \sin \omega t d t=I_{4}^{+}=\frac{\pi \omega^{2}}{\beta \sinh \left(\frac{\pi \omega}{2 \sqrt{\alpha}}\right)}
\end{aligned}
$$

Thus, $M^{-}\left(t_{0}\right)$ has simple zeros and chaotic motions occur if and only if

$$
\frac{\mu}{f}<\left|\frac{I_{3}^{-}+I_{4}^{-}}{I_{1}^{-}-I_{2}^{-}}\right|
$$

Now we discuss the chaotic feature on the system parameters. First, taking $\alpha=1, \beta=0.1,0.2, \cdots, 0.7$, respectively, the critical curves of system (2) for $\Gamma+$ in $(\mu / f, \omega)$ plane are shown as in Fig 2(a). The critical curve has a classical bell shape in this case. This means that, with the excitations possessing sufficiently small or very large periods, the systems are not chaotically excited. One can also see that for fixed $\omega$, the critical value for chaos first increases and then 
decreases as the increasing of $\beta$. Next, taking $\beta=1, \alpha=0.1,0.2, \cdots \quad 0.7$, respectively, the critical curves for chaotic motions of system (2) for $\Gamma+$ are shown as in Fig 2(b). From Fig 2(b) one can see that for fixed small values of $\omega$, the critical values of chaotic motions decrease monotonously as the increase of $\alpha$; but the case is opposite for fixed large values of $\omega$. Last, for $\alpha=1, \beta=0.1$ or $\beta=1$, the critical curves of chaos (for $\Gamma^{+}$) for systems with periodically parametric excitation or external excitationare shown as in Fig. 3. From Fig. 3 we can see that the critical value of systems with parametric excitations is larger than that of systems with external excitations. When $\alpha=1$, the critical curves of system (1) for $\Gamma^{-}$in $(\mu / f, \omega)$ plane are shown as in Fig 4(a). From Fig 4(a) we can see that when $\alpha \geq 0.3$, the critical curve first decreases quickly to zero and then increases, at last it decreases to zero as $\omega$ increases from zero. There exists a controllable frequency $\omega$ excited at which chaotic motions do not take place no matter how large the excitation amplitude is. When $\alpha$ is fixed, the controllable frequency increases as $\beta$ increases. While $\beta=1$, the critical curves for chaotic motions of system (1) for $\Gamma^{-}$are shown as in Fig. 4(b). From Fig. 4 (b) we can see that there also exists a controllable frequency which is near 1.4 and decreases as $\alpha$ increases. For $\alpha=1, \beta=1$ or $\beta=0.1$, the critical curves of chaos (for $\Gamma^{-}$) for systems with periodically parametric excitation or external excitation are shown as in Fig. 5. From Fig. 5 we can see that for $\beta=0.1$, when the frequency $\omega$ is small, the critical value of systems with external excitations is larger than that of systems with parametric excitations; while $\omega$ crosses a critical value, the critical value of systems with external excitations is smaller than that of systems with parametric excitations. While for $\beta=1$ the case is opposite. When $\alpha=1, \beta=1$, or $\beta=0.1$ the critical curves of system (1) for $\Gamma^{+}$and $\Gamma^{-}$are shown as in Fig. 6 . From Fig. 6 we can see that the critical value of is smaller than that of $\Gamma^{+}$, which means as the increase of the excitation amplitude, $\Gamma^{-}$is first chaotically excited, and then both $\Gamma^{+}$and $\Gamma^{-}$are chaotically excited.

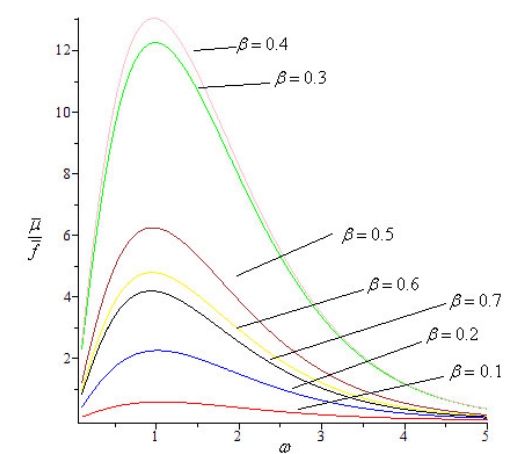

(a)

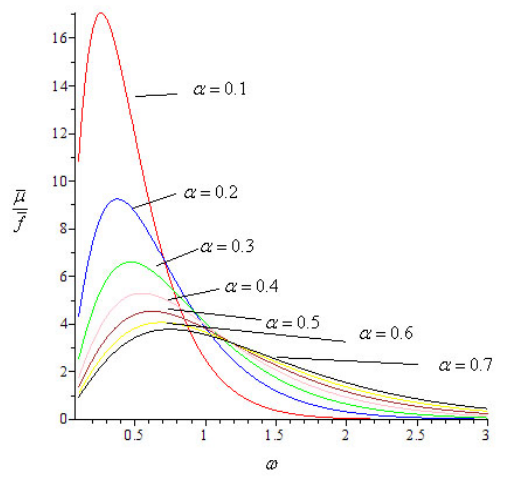

(b)

Fig. 2 The critical curve for different values of (a) $\beta$, (b) $\alpha$ for $\Gamma^{+}$

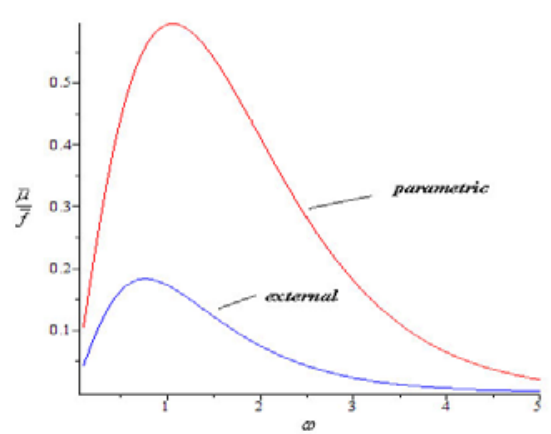

(a)

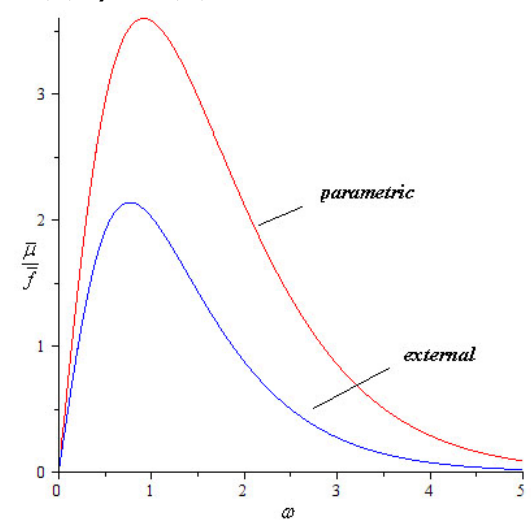

(b)

Fig.3 The critical curves of $\Gamma^{+}$for (a) $\alpha=1, \beta=0.1$, (b) $\alpha=1, \beta=1$ 


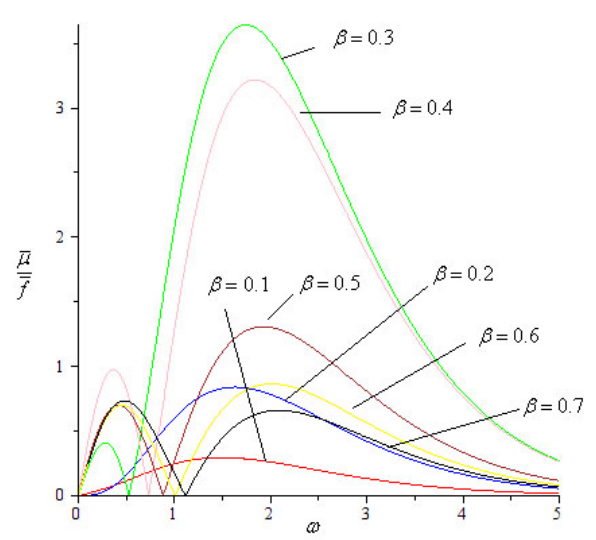

(a)

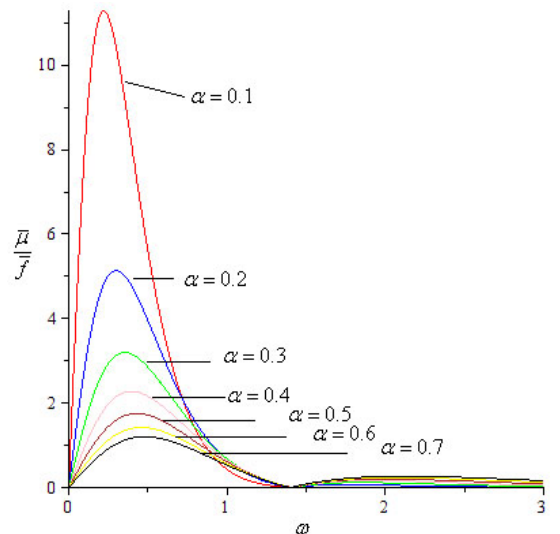

(b)

Fig.4 The critical curve for different values of (a) $\beta$, (b) $\alpha$ for $\Gamma^{-}$.

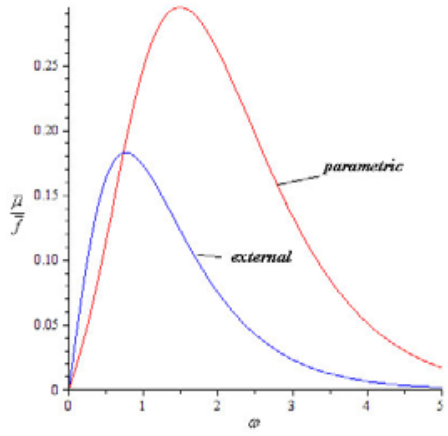

(a)

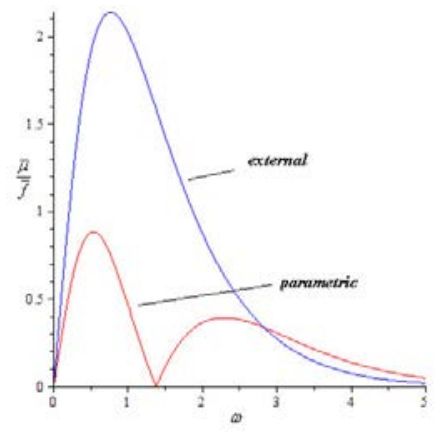

(b)

Fig.5 The critical curves of $\Gamma^{+}$and $\Gamma^{-}$for $(a) \alpha=1, \beta=0.1$, (b) $\alpha=1, \beta=1$.

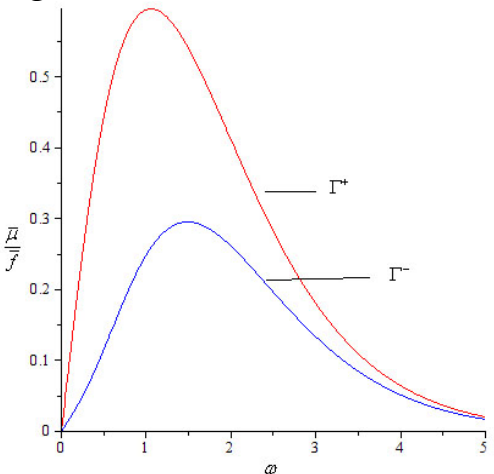

(a)

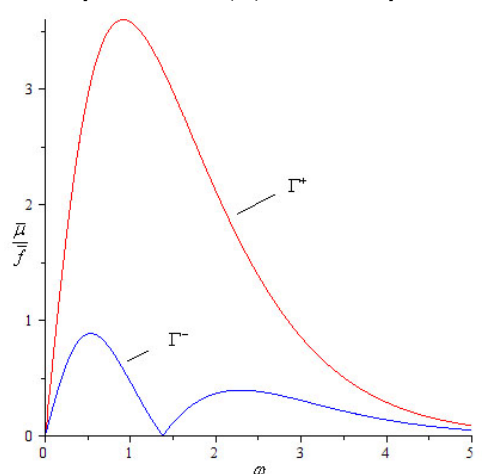

(b)

Fig. 6 The critical curves of $\Gamma^{-}$for (a) $\alpha=1, \beta=0.1$, (b) $\alpha=1, \beta=1$.

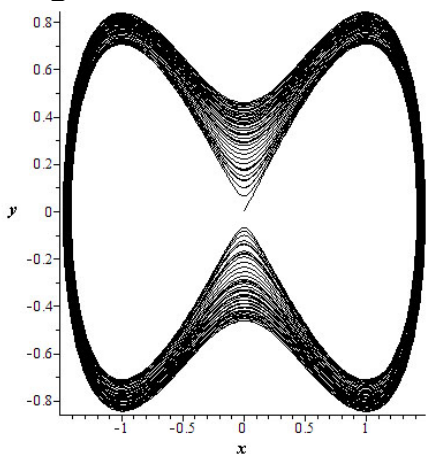

(a)

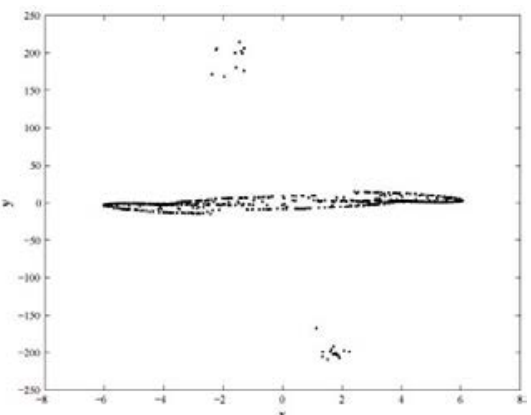

(b)

Fig.7 The (a) phase portraits and (b) Poincar'e sections of system (2) for $\alpha=\beta=1$. 


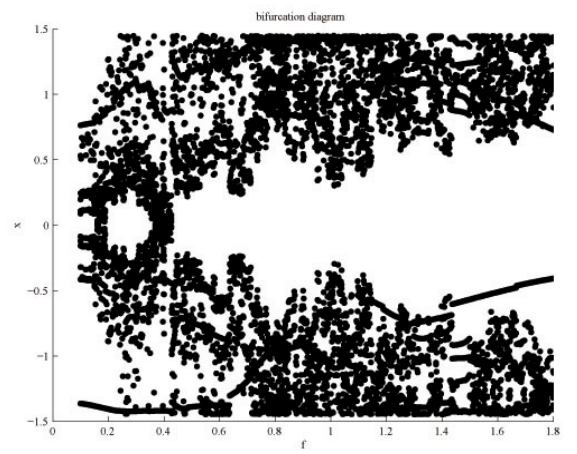

Fig.8 The bifurcation diagram of system (2) for $\alpha=\beta=1$.

\section{Numerical simulations}

Using the fourth-order Runge-Kutta method, choosing the system parameters $\varepsilon=0.001, \bar{f}=\bar{\mu}=1, \omega=1, \alpha=1, \beta=1$, the initial value $(x(0), y(0))=(0.001,0.001)$, the phase portrait and Poincaré section are shown as in Fig .7. Noting that these parameters are in the chaotic region, so the system is chaotic excited, which agree with the analytical results. For $f \in[0.1,1.8]$, the bifurcation diagram of system (2) is shown as in Fig. 8, from which we can see that paroxysmal chaos will occur as the increasing of the excitation amplitude.

\section{Conclusions}

Using the Melnikov method, chaotic motions for a Rayleigh-Duffing oscillator with periodically external and parametric excitations are investigated in the cases of double-well potential. The chaotic feature on the system parameters is discussed in detail. It is presented that there exist a "controllable frequency" for this system. Numerical simulations verify the analytical results, and also present that paroxysmal chaos will occur as the increasing of the excitation amplitude.

\section{References}

[1] Felix, J.L.P., Balthazar, J.M., Brasil, R.M.L.R.F., 2009. Comments on nonlinear dynamics of a non-ideal Duffing-Rayleigh oscillator: numerical and analytical approaches. Journal of Sound and Vibration, 319, 1136-1149.

[2] Kanai Y., Yabuno H., 2012. Creation-annihilation process of limit cycles in the Rayleigh-Duffing oscillator. Nonlinear Dynamics, 70, 1007-1016.

[3] Qriouet M.T., Mira C., 2000. Reducible fractional harmonics generated by the non-autonomous Duffing-Rayleigh equation. Pockets of reducible harmonics and Arnold's tongues. International Journal of Bifurcation and Chaos, 10, 1345-1366.

[4] Xie W.X., Xu W., L. Cai, 2005. Path integration of the Duffing-Rayleigh oscillator subject to harmonic and stochastic excitations, Applied Mathematics and Computation, 171, 870-884.

[5]Xie W.X., Xu W., L. Cai, 2006. Study of the Duffing-Rayleigh oscillator subject to harmonic and stochastic excitations by path integration, Applied Mathematics and Computation, 172, 1212-1224.

[6] Siewe S.M., Tchawoua C., Rajasekar S., 2012. Parametric resonance in the RayleighDuffing oscillator with time-delayed feedback. Communications in Nonlinear Science \& Numerical Simulation, 17, 4485-4493.

[7] Mihara T., Kawakami, H., 1996. Synchronization and chaos of coupled DuffingRayleigh oscillators, IEEE Transactions on Fundamentals of Electronics. Communications and Computer Sciences, E79-A, 1581-1586. 
[8] Siewe S.M., Cao H.J., Sanjuan M.A.F., 2009. Effect of nonlinear dissipation on the basin boundaries of a driven two-well Rayleigh-Duffing oscillator. Chaos, Solitons and Fractals, 39, 1092-1099.

[9] Siewe S.M., Cao H.J., Sanjuan M.A.F., 2009. Effect of nonlinear dissipation on the basin boundaries of a driven two-well Rayleigh-Duffing oscillator. Chaos, Solitons and Fractals, 39, 1092-1099.

[10] Siewe S.M., Tchawoua C., Woafo P., 2010. Melnikov chaos in a periodically driven Rayleigh-Duffing oscillator, Mechanics Research Communications,37, 363-368.

[11] Zhang Y.L., Luo M.K., 2012. Fractional Rayleigh-Duffing-like system and its synchronization. Nonlinear Dynamics, 70, 1173-1183.

[12] Miwadinou C. H., Monwanou A. V., Chabi Orou J. B., 2015. Effect of Nonlinear Dissipation on the Basin Boundaries of a Driven Two-Well Modified RayleighDuffing Oscillator. International Journal of bifurcation and Chaos, 25, 1550024.

[13] Zhang R.R., Xu W., Yang G.D., et al. 2015. Response of a Duffing Rayleigh system with a fractional derivative under Gaussian white noise excitation, Chinese Physics B, 24, 020204.

[14] Yue X.L., Xu W., Zhang Y., 2012. Global bifurcation analysis of Rayleigh-Duffing oscillator through the composite cell coordinate system method, Nonlinear Dynamics, 69, 437-457.

[15] Wiggins S., 1990. Introduction to applied non-linear dynamical systems and chaos, Springer, New York. 